\title{
Reciprocal Effects of Social Support in Major Depression Epidemiology
}

\author{
Scott B. Patten*, Jeanne V.A. Williams, Dina H. Lavorato and Andrew G.M. Bulloch \\ Department of Community Health Sciences, Faculty of Medicine, University of Calgary, $3^{\text {rd }}$ Floor TRW Building, 3280 \\ Hospital Drive NW, Calgary, Alberta, Canada, T2N 4Z6
}

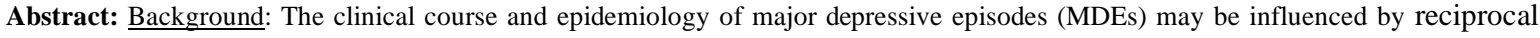
interactions between an individual and the social environment. Epidemiological data concerning these interactions may assist with anticipating the clinical needs of depressed patients. Methods: The data source for this study was a Canadian longitudinal study, the National Population Health Survey (NPHS), which provided 8 years of follow-up data. The NPHS interview included a brief diagnostic indicator for MDE, the Composite International Diagnostic Interview Short Form for Major Depression (CIDI-SFMD). The NPHS interview also incorporated the Medical Outcomes Study Social Support Scale (MOSSS) and a set of relevant demographic and health-related measures. The MOSSS assesses total social support and four specific dimensions of social support. Hazard ratios (HR) were used to quantify associations in the longitudinal data. Results: Lower quartile total social support ratings predicted MDE incidence: the HR adjusted for age and sex was 1.9 (95\% CI 1.6 - 2.2). Lower quartile ratings in specific social support dimensions yielded similar HRs. MDE was associated with emergence of lower-quartile affection social support (age and sex adjusted HR 1.3, 95\% CI 1.1 - 1.7), but other aspects of social support were not consistently associated with MDE. Conclusions: Low social support appears to be a robust risk factor for MDE and can be used to identify persons at higher risk of MDE. Evidence that MDE has a negative effect on social support was weaker and was restricted to affection social support.
\end{abstract}

Keywords: Major Depressive Disorder, Mood Disorder, Epidemiology, Longitudinal Studies, Risk Factors.

\section{INTRODUCTION}

The epidemiology of major depressive episode (MDE) may involve reciprocal interactions with the social environment, including social support. Low social support is a predictor of MDE risk [1], and a determinant of prognosis [2]. Furthermore, MDE may influence the risk of low social support. Rohde et al. reported that social skills diminish after an episode of depression in people aged 50+ [3]. This may, in turn lead to diminished social support. Similarly, Leskelä et al. reported that both objective and subjective ratings of social support declined during persistent MDE [4]. A related literature is concerned with the possibility of "scar effects" in association with MDE. A scar effect is a negative psychological change associated with depression that persists after an episode has resolved. Studies concerned with scar effects have generally failed to identify long-term changes in social support that meet this definition [5,6]. A broader and perhaps more applicable concept is that of an "erosive" effect, as articulated by Joiner [7]. If depression changes perceptions of social support, this may lead to counterproductive strategies such as negative feedback-seeking and excessive reassurance-seeking. Such strategies may, in turn, lead to rejection and/or isolation.

An extensive literature has examined etiological aspects of the relationship between social support and mental health outcomes, providing evidence both for favourable direct effects of social support and providing support for the idea that

*Address correspondence to this author at the Department of Community Health Sciences, Faculty of Medicine, University of Calgary, $3^{\text {rd }}$ Floor TRW Building, 3280 Hospital Drive NW, Calgary, Alberta, Canada. T2N 4 Z6. Tel: 1.403.220.8734, Fax: 1.403.270.7307; Email: patten@ucalgary.ca social support may buffer the negative impact of stressful life events $[8,9]$. Because of the dynamic nature of these relationships, specialized studies involving frequent and detailed assessments are likely to be required to understand its etiological characteristics. However, epidemiologic studies can make a contribution by examining changes over long periods of time in representative populations. Epidemiological description of the long-term impact MDE on social support, and vice versa, can help to define the implications of these relationships for clinical care and for the organization of services. For example, if low social support is associated with a higher risk of MDE, this may have implications for primary, secondary and tertiary prevention. Provision of social support through formal programs (e.g. therapist-led support groups, peer-support initiatives) may help to prevent MDE (primary prevention). Similarly, higher "base-rate" risks of MDE in populations with low social support may lead to enhanced positive predictive values for screening instruments and a greater efficiency of screening (higher positive predictive values for tests with specific sensitivity and specificities) in these populations. Finally, interventions to improve social support may reduce relapse rates in established cases and may also counteract negative changes that would otherwise occur to social support (tertiary prevention). The goal of this study was to examine the epidemiologic relationship between MDE and social support in a large, representative cohort within the general population.

\section{MATERIALS AND METHODOLOGY}

The data source for this analysis was the National Population Health Survey (NPHS), a longitudinal study based on a nationally representative community sample assembled by 
Statistics Canada (Canada's national statistical agency) in 1994. However, the current study was based on data collected starting in 1998 when a validated social support measure was first included in the NPHS interview. Detailed information about NPHS methods may be found on the Statistics Canada Web page [10]. Because social support may affect the prognosis of depression [2], our strategy was to focus on the new occurrence of health events or transitions by examining incidence rather than prevalence. The goal was to prevent the confounding of risk with prognosis, as occurs when prevalence is the unit of measure. This incidence-based approach requires the identification of populations at risk for the relevant health transitions: (1) a cohort without MDE at the baseline interview, who are at risk of incident MDE, and (2) a cohort with adequate social support at the baseline time point, who are at risk for transition to low social support.

Assessment of MDE in the NPHS is based on the Composite International Diagnostic Interview Short Form for Major Depression (CIDI-SFMD) [11], which assesses past year MDE. The CIDI-SFMD is scored with a predictive probability algorithm based on the number of symptombased criteria fulfilled during a 2 -week period in the year preceding the interview. The instrument was scored at the $90 \%$ predictive probability level in this analysis, indicating endorsement of five symptoms resembling the DSM-IV ' $A$ ' criterion for MDE [12]. Consistent with DSM-IV, the scoring algorithm stipulates that at least one of these symptoms must be depressed mood or loss of interest or pleasure.

The scale used to assess social support in the NPHS is the Medical Outcomes Study Social Support Scale (MOSSS) [13]. This scale was developed using an analysis of ratings obtained from 2987 participants in the Medical Outcomes Study. From an initial pool of 50 candidate items, 19 were selected for inclusion in the scale. The MOSSS is a functional social support measure, as distinct from a structural one. MOSSS items assess four functional domains of social support: informational/emotional support, tangible support, positive social interaction and affection support. In early stages of scale development, informational and emotional social supports were conceptualized separately, but psychometric analyses indicated inadequate distinction between these dimensions. As a result, they were collapsed into a single subscale. Additional analyses indicated that calculation of an overall index of social support was justifiable [13].

The MOSSS category informational/emotional social support refers to aspects of communication between a respondent and someone to whom that respondent feels close. In addition to this subscale and the total social support score, the MOSSS evaluates three other subscales. Tangible support refers to material support or aid. Positive interaction refers to the concepts of integration, belonging and social companionship. Affection social support emphasizes behavioural manifestations and consists of three items: having "someone who hugs you", "someone to love you and make you feel wanted" and "someone who shows you love and affection" [13].

The NPHS longitudinal cohort included 17,276 participants, but the current analysis was restricted to $n=13,529$ respondents who were 12 years or older at the time of the 1998 interview when the MOSSS was first used. Further, the analysis was restricted to subsets of the NPHS cohort who were at risk of MDE (in the part of the analysis concerned with MDE risk) and of low social support (in the parts of the analysis concerned with transitions to lower levels of social support). Since the social support status of these respondents may have been influenced by prior episodes of MDE, this exclusion would ideally have been applied to all respondents with any lifetime history of MDE, however, lifetime history was not assessed during early cycles of the NPHS.

In the part of the analysis examining the effect of social support on MDE risk, the analysis was restricted to those who did not have an episode of MDE in the year preceding the 1998 interview. In the part of the analysis concerned with an effect of MDE on the risk of developing low social support, the analysis was restricted to respondents who did not have low scores for social support in 1998, with "low" being defined as lower quartile ratings on the MOSSS scale and subscales. The NPHS cohort has been interviewed every two years and data are available up to 2006. Fig. (1) summarizes loss to follow-up in the NPHS cohort between 1998 and 2006. The 1994 NPHS interviews were mostly conducted face to face, but almost all of the follow-up interviews have been conducted over the telephone, including all of the estimates for the 1998 to 2006 interval relevant to this study.

Initially, the incidence of MDE was tabulated against the various social support indices in the first interview cycle after the initial application of the MOSSS in 1998 (the first subsequent cycle was 2000). Respondents with MDE in 1998 were excluded so that MDE detected in 2000 would represent new (incident) episodes. Estimation of incidence was then extended to subsequent follow-up using discrete time proportional hazard models, as described below. These models also allowed an estimate to be made of the unadjusted and adjusted HRs for the effect of social support on MDE and that of MDE on social support.

The effect of low social support on MDE risk (and vice versa) was evaluated using proportional hazard models. Because the NPHS employed interviews at discrete (2 year) intervals, a model for grouped time data was used. The model was a generalized linear model of the binomial family using a complementary log-log link function. Jenkins has outlined procedures for implementation of such analyses in STATA [14]. The models presented here are non-parametric: time intervals are represented using indicator variables with no assumptions being made about the pattern of change in risk over time. Cross-product interaction terms between MDE or social support and the indicator variables for time were used to test the proportional hazard assumption (using a $\log$ rank test). No violations of the assumption were identified. Respondents developing the outcome (lack of social support or MDE, depending on the part of the analysis), dying or who were lost to follow-up or institutionalized were censored from the models at subsequent time points but their data up to that time were included in the analysis. The exposure variables (MDE and lower quartile social support) were treated as time-varying characteristics in the analysis, such that the respondents' status at each cycle was treated as a determinant of risk at the subsequent cycle. In the part of the analysis concerned with low social support as a risk factor for MDE, the following variables were included as covari- 


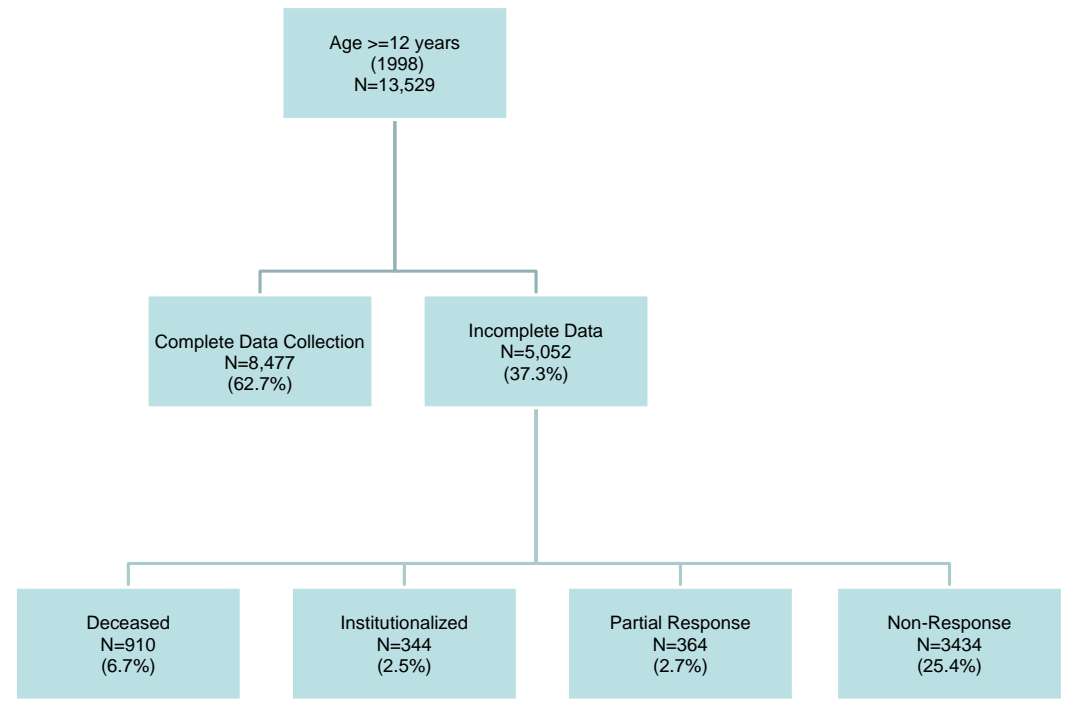

Fig. (1). Attrition from the NPHS Cohort 1998-2006.

ates: marital status, employment and level of education. These variables were measured in the NPHS using standard questionnaire items [10].

The NPHS used a multistage, stratified design that included clustering to select eligible households. To adjust for design effects, Statistics Canada recommends sampling weights and a bootstrap procedure that uses a set of 500 replicate sampling weights. This procedure has been used in all analyses reported here. The study received approval from the University of Calgary Conjoint Ethics Board.

\section{RESULTS}

In analyses concerned with low social support as a risk factor for MDE, individuals with MDE in 1998 were initially identified $(n=579)$ and excluded from subsequent analysis, leaving a sample size of $n=12,351$ that could be considered at risk. Demographic features of this subset of the sample are presented in Table 1. Incident MDE was more common in women than men and declined with age after the age of 45. The low incidence of MDE in divorced, separated or widowed respondents is probably due to confounding by age, since depression incidence declines with age and this is likely to be an older group.

The overall incidence of MDE, which was $3.7 \%$ (95\% CI $3.3-4.1)$ in the 1998 to 2000 interval, then declined slightly in subsequent cycles to $2.2 \%(95 \%$ CI $1.8-2.7)$ in the 2004 to 2006 interval. This reflects an expected diminishing risk of developing a new MDE with increasing duration of time without an MDE. Respondents with lower quartile total social support ratings had a higher incidence of MDE in the initial 1998 to 2000 interval: $5.1 \%$ (95\% CI 4.1 - 6.1), compared to $3.3 \%(95 \% 2.9-3.8)$ in the group with higher levels of support. There was no evidence of an association between marital status, education level nor employment status on MDE incidence. Age and sex were strongly associated with incident MDE in all analyses. The age and sex adjusted HR for lower quartile total social support was 1.9 (95\% CI 1.6-2.2), indicating an approximate doubling of risk in those with low social support. Age and sex adjusted HRs for the MOSSS social support subtypes are presented in Table 2 . In the right hand column, adjustments are also made for marital status, education and employment status. Consistent associations of MDE with each of the MOSSS subscales were observed.

In the part of the analysis assessing the impact of MDE on social support transitions $n=3,269$ respondents were excluded from the analysis because of low social support ratings at the baseline time point, leaving $n=9,340$ eligible respondents for this part of the analysis. Within this group, $3.2 \%$ (95\% CI 2.7-3.7) had an MDE episode detected in the 1998 interview. In this depressed group, $22.5 \%$ (95\% CI 16.6-28.4) had a lower quartile total social support score two years later in the 2000 interview, slightly higher than the $16.9 \%$ (95\% CI 15.8-18.0) incidence of low total social support among those without an MDE episode in the year preceding the 1998 interview. Table 3 shows the incidence of low social support in this initial 1998 to 2000 interval in each of the MOS subscales of social support.

Proportional hazard models evaluating the impact of MDE on transitions to low social support identified interactions between MDE, age group and marital status in analyses concerned with total social support, tangible support and positive social interaction (all $\mathrm{p}$ values $\leq 0.05$ ). These interactions suggested a lower degree of impact of MDE on transition to lower quartile social support in single (never married) respondents and in the middle age ranges (19 to 25 and 26 to 65 ). Normally, the presence of such interactions would necessitate reporting of age and marital status specific HRs, however, due to sample size constraints these stratum specific estimates were imprecise and did not achieve statistical significance. For this reason, HRs are not reported for these outcomes in Table 3. For emotional/informational support there were no statistically significant interactions, but the HR did not achieve statistical significance, see Table 3 . The analysis focusing on low affection social support found no significant interactions between $\mathrm{MDE}$ and any other variable. The unadjusted HR for MDE was 1.3 (95\% CI 1.1 1.7), only slightly higher than that for emotional/informational support, but which was statistically significant $(\mathrm{p}=0.013)$. 
Table 1. Demographic Features and 2-Year Incidence* of MDE among Non-Depressed** Members of the NPHS Longitudinal Cohort in 1998-2000

\begin{tabular}{|c|c|c|}
\hline & $\begin{array}{c}\text { Frequency (\%) } \\
95 \% \text { CI } \\
\text { n=12,351 }\end{array}$ & $\begin{array}{c}\text { Incidence }(\%) * \\
95 \% \mathrm{CI} \\
\mathrm{n}=\mathbf{4 1 8}\end{array}$ \\
\hline Male & $\begin{array}{c}49.4 \\
48.8-50.1\end{array}$ & $\begin{array}{c}2.8 \\
2.2-3.4\end{array}$ \\
\hline Female & $\begin{array}{c}50.6 \\
49.9-51.2\end{array}$ & $\begin{array}{c}4.5 \\
3.9-5.2\end{array}$ \\
\hline Age $12-18$ & $\begin{array}{c}11.1 \\
10.3-11.8\end{array}$ & $\begin{array}{c}4.9 \\
3.0-6.7\end{array}$ \\
\hline Age $19-25$ & $\begin{array}{c}10.8 \\
10.2-11.5\end{array}$ & $\begin{array}{c}4.4 \\
2.8-5.9\end{array}$ \\
\hline Age $26-45$ & $\begin{array}{c}37.7 \\
37.0-38.5\end{array}$ & $\begin{array}{c}4.4 \\
3.6-5.1\end{array}$ \\
\hline Age $46-65$ & $\begin{array}{c}26.6 \\
25.9-27.3\end{array}$ & $\begin{array}{c}3.1 \\
2.4-3.8\end{array}$ \\
\hline Age $\geq 66$ & $\begin{array}{c}13.8 \\
13.3-14.2\end{array}$ & $\begin{array}{c}1.2 \\
0.5-1.9\end{array}$ \\
\hline Secondary level or less & $\begin{array}{c}43.0 \\
41.9-44.2\end{array}$ & $\begin{array}{c}3.8 \\
3.1-4.5\end{array}$ \\
\hline Some education above secondary school & $\begin{array}{c}57.0 \\
55.8-58.1\end{array}$ & $\begin{array}{c}3.6 \\
3.0-4.2\end{array}$ \\
\hline Married/Common-law & $\begin{array}{c}57.3 \\
56.3-58.4\end{array}$ & $\begin{array}{c}3.3 \\
2.8-3.9\end{array}$ \\
\hline Single & $\begin{array}{c}29.3 \\
28.4-30.3\end{array}$ & $\begin{array}{c}4.6 \\
3.7-5.6\end{array}$ \\
\hline Widowed/separated/divorced & $\begin{array}{c}13.3 \\
12.6-14.0\end{array}$ & $\begin{array}{c}3.2 \\
2.3-4.1\end{array}$ \\
\hline Currently Employed/Student & $\begin{array}{c}70.9 \\
70.0-71.8\end{array}$ & $\begin{array}{c}3.9 \\
3.3-4.5\end{array}$ \\
\hline Not Currently Employed/Not Student & $\begin{array}{c}29.1 \\
28.2-30.0\end{array}$ & $\begin{array}{c}3.1 \\
2.5-3.8\end{array}$ \\
\hline
\end{tabular}

* an episode identified by the CIDI-SFMD in the year preceding the year 2000 interview. ** no MDE episode in the year preceding the 1998 interview.

\section{DISCUSSION}

These results provide firm evidence that low social support is associated with an increased risk of MDE. This amounts to a replication of earlier studies examining the predictive value of social support for MDE risk [1]. All of the indices of social support examined were strong predictors of new onset MDE in NPHS respondents. These results highlight the possibility that provision of social support to those with low support may contribute to primary prevention of MDE. Because of these higher risks, the positive predictive value of depression screening tests is likely to be higher in those with low levels of social support. Finally, as many of the new episodes of MDE observed in this study can be assumed to represent recurrences, provision of social support may be valuable for reducing the morbidity associated with MDE. However, the actually effectiveness and costeffectiveness of primary, secondary and tertiary interventions require evaluation prior to drawing any firm conclusions.

Effects in the other direction (MDE as a determinant of transition to lower social support categories) were inconsistent. The strongest evidence was for affection social support. This category of social support emphasizes behavioural manifestations of affection, such as showing love and affec- 
Table 2. Age and Sex Adjusted HRs for the Four MOSSS Social Support Dimensions

\begin{tabular}{|c|c|c|c|}
\hline & $\begin{array}{c}\text { Unadjusted HR } \\
\mathbf{( 9 5 \%} \mathbf{~ C I )}\end{array}$ & $\begin{array}{c}\text { Age and Sex Adjusted HR (95\% } \\
\text { CI) }\end{array}$ & $\begin{array}{c}\text { Age, Sex, Marital Status, } \\
\text { Education, Employment } \\
\text { Adjusted HR (95\% CI) }\end{array}$ \\
\hline \hline Emotional or Informational Support* & 1.6 & 1.7 & $\begin{array}{c}1.7 \\
1.5-2.0\end{array}$ \\
\hline Tangible Support* & $1.3-1.8$ & $1.5-2.0$ & 1.5 \\
\hline Positive Social Interaction* & 1.5 & 1.6 & $1.3-1.8$ \\
\hline Affection Social Support* & $1.3-1.8$ & 1.7 & 1.7 \\
& 1.6 & $1.4-2.0$ & $1.4-2.0$ \\
\hline
\end{tabular}

* lower quartile subscale scores.

Table 3. Incidence of Low Social Support 1998 to 2000 and in Proportional Hazard Models for the MOS Subscales

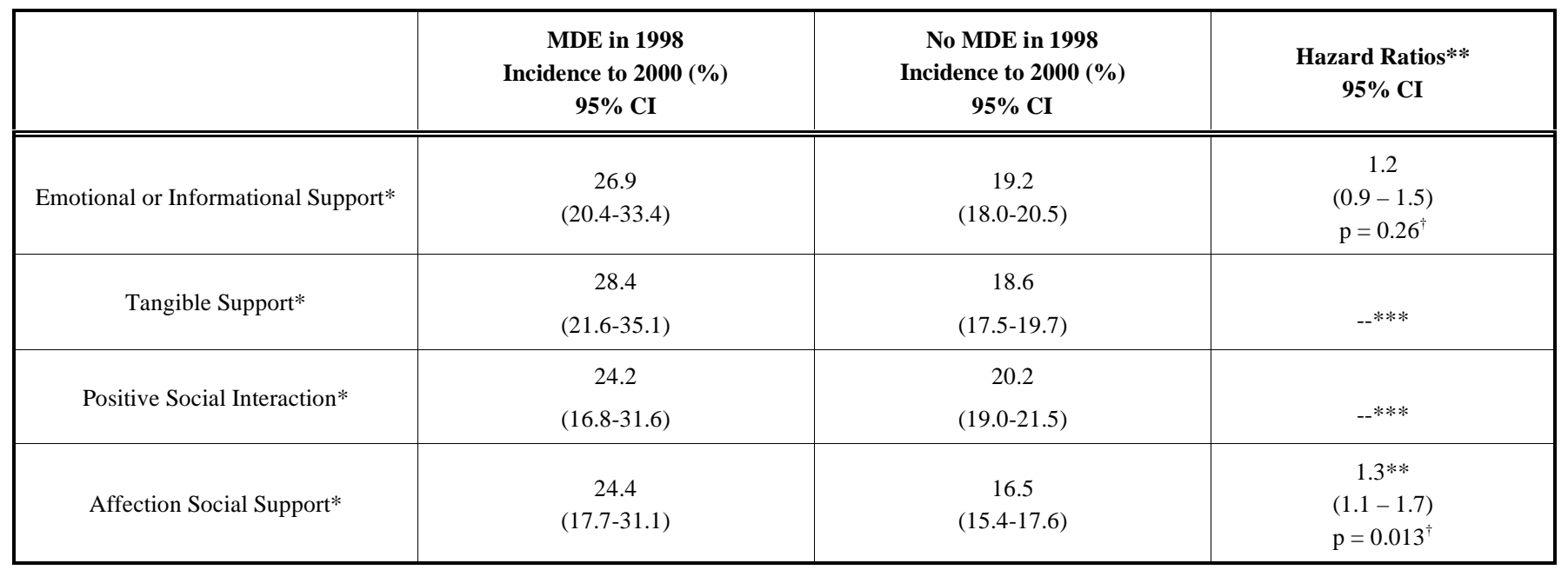

* lower quartile subscale scores.

** adjusted for age, sex, education, marital status.

*** HR not reported because of interactions with age and marital status (see text)

$\dagger$ Wald test, bootstrapped.

tion, or otherwise making a person feel loved and wanted. The results seen for affection social support require replication, but the result implies that more intimate types of relationships, those that involve expression of affection, are more vulnerable to disruption by MDE. While awareness of this reality is likely to be valuable in clinical practice, affection social support may be less amenable to formal intervention than other dimensions of social support. Nevertheless, the results emphasize the value of having the capability in clinical settings to assess and support relevant relationships, such as family and marital relationships.

The results of this analysis do not indicate that MDE is free of adverse repercussions on other dimensions of social support. However, the effects on dimensions other than affection social support appear to be less consistent, with interactions evident across age and marital status categories. More detailed research approaches will be needed to more fully define the erosive effects of MDE on various aspects of social support.
The study has several limitations. The CIDI-SFMD is a brief instrument that does not include the detailed symptom probes of the full CIDI interview. Available evidence suggests that it may be somewhat non-specific [15]. Consistent with this idea, studies using the CIDI-SFMD have typically produced slightly higher estimates than studies using the full CIDI. The MOSSS can also be criticised because of its combination of two social support functions: information and emotional support, into a single subscale and also by its lack of coverage of self-esteem or self-appraisal support. The developers of the scale were of the opinion that this type of support (a positive comparison between oneself and others) was less important in the chronically ill population targeted during development of the scale [13]. Another limitation of this study is the lengthy period of time between the NPHS interviews, which meant that the analysis could only address large changes evident over the long term. The etiologic interplay between social support and MDE may unfold in important ways over a shorter-term time course in association with major life events. The NPHS interviews were con- 
ducted two years apart, yet the CIDI-SFMD only covers past year depressive episodes. Therefore, unobserved changes may have occurred between the NPHS interviews. The selection of the lower quartile as an indicator of low social support is also somewhat arbitrary.

Many new onset MDEs were detected in the younger age categories, and MDE incidence declined with age in this analysis. This pattern is consistent with that observed in prior studies of MDE incidence in Canada (which have all used the NPHS data) [16-18] and also consistent with the pattern observed in longitudinal studies of youth conducted elsewhere in North America.

\section{CONCLUSIONS}

This study found that low social support in all of the assessed dimensions was a predictor of MDE risk. This finding supports the idea that provision of social support may contribute to prevention of MDE. Less consistent evidence of an effect of MDE on social support was found. However, a statistically significant increase in the risk of transition to lower affection social support was observed in depressed respondents. Additional studies are needed to replicate the latter finding. If replicated, this result may point towards a specific vulnerability in depressed persons and could lead greater emphasis on interventions designed to prevent or minimize diminished affection in the social networks of depressed persons.

\section{LIST OF ABBREVIATIONS}

$\begin{array}{ll}\text { CIDI } & \begin{array}{l}\text { Composite International Diagnostic } \\ \text { Interview }\end{array} \\ \text { CIDI-SFMD }=\begin{array}{l}\text { Composite International Diagnostic } \\ \text { Interview }- \text { Short Form for Major De- } \\ \text { pression }\end{array} & \begin{array}{l}\text { Diagnostic and Statistical Manual of } \\ \text { Mental Disorders, } 4^{\text {th }} \text { Edition }\end{array} \\ \text { DSM-IV } & =\begin{array}{l}\text { Major Depressive Episode } \\ \text { MOSSS }\end{array} \\ \text { NPHS } & =\begin{array}{l}\text { Medical Outcomes Study Social Sup- } \\ \text { National Population Health Survey }\end{array} \\ \text { CONFLICTS OF INTEREST }\end{array}$

The authors have no conflicts of interest relative to this manuscript. Dr. Patten has received speaking and consulting fees from Cipher Pharmaceuticals, Lundbeck and Servier Canada.

\section{ACKNOWLEDGEMENT AND DISCLAIMER}

Dr. Patten is a Senior Health Scholar with the Alberta Heritage Foundation for Medical Research. Dr. Bulloch and
Patten are members of the Hotchkiss Brain Institute. This project was funded by a grant from the Canadian Institutes of Health Research. This analysis is based on data collected by Statistics Canada. However, the results do not reflect the opinions of Statistics Canada.

\section{REFERENCES}

[1] Bottomley C, Nazareth I, Torres-González F, Svab I, Maaroos H-I, Geerlings MI, et al. Comparison of risk factors for the onset and maintenance of depression. Br J Psychiatry 2010; 196: 13-7.

[2] Spijker J, de Graaf R, Bijl RV, Beekman ATF, Ormel J, Nolen WA. Determinants of persistence of major depressive episodes in the general population. Results from the Netherlands Mental Health Survey and Incidence Study (NEMESIS). J Affect Disord 2004; 81: 231-40.

[3] Rohde P, Lewinsohn PM, Seeley JR. Are people changed by the experience of having an episode of depression? A further test of the scar hypothesis. J Abn Psychol 1990; 99: 264-71.

[4] Leskela U, Melartin T, Rytsala H, Sokero P, Lestela-Mielonen P, Isometsa $\mathrm{E}$. The influence of major depressive disorder on objective and subjective social support: a prospective study. J Nerv Ment Dis 2008;196(12): 876-83.

[5] Beevers CG, Rohde P, Stice E, Nolen-Hoeksema S. Recovery from major depressive disorder among female adolescents: a prospective test of the scar hypothesis. J Consult Clin Psychol 2007; 75(6): 888-900.

[6] Zeiss AM, Lewinsohn PM. Enduring deficits after remissions of depression: a test of the scar hypothesis. Behav Res Ther 1988; 26(2): $151-8$

[7] Joiner TE. Depression's vicious scree: Self-propogating and erosive processes in depression chronicity. Clin Psychol Sci Pract 2000; 150: 720-7.

[8] Cohen S, Wills TA. Stress, social support, and the buffering hypothesis. Psychol Bull 1985; 98(2): 310-57.

[9] Dalgard OS, Bjork S, Tambs K. Social support, negative life events and mental health. Br J Psychiatry 1995; 166(1): 29-34.

[10] Statistics Canada National Population Health Survey Household Component Cycle 1 to 7 (1994/1995 to 2006/2007) longitudinal documentation. Ottawa: Statistics Canada; 2009. Report No.: 3225.

[11] Kessler RC, Andrews G, Mroczek D, Ustun B, Wittchen HU. The world health organization composite international diagnostic interview short-form (CIDI-SF). Int J Methods Psychiatr Res 1998; 7: 171-85.

[12] American Psychiatric Association. Diagnostic and Statistical Manual of Mental Disorders (DSM-IV-TR). Washington: American Psychiatric Association; 2000.

[13] Sherbourne CD, Stewart AL. The MOS Social Support Survey. Soc Sci Med 1991; 32: 705-14.

[14] Jenkins SP. Discrete time proportional hazards regression. STATA Tech Bull 1997; STB-39: 22-31.

[15] Patten SB, Brandon-Christie J, Devji J, Sedmak B. Performance of the Composite International Diagnostic Interview Short Form for Major Depression in a community sample. Chron Dis Can 2000; 21: 68-72.

[16] Wang JL, Williams JVA, Lavorato DH, Schmitz N, Dewa C, Patten SB. The incidence of major depression in Canada: The National Population Health Survey. J Affect Disord 2010; 123: 158-63.

[17] Patten SB. Incidence of major depression in Canada. CMAJ 2000 19; 163(6): 714-5.

[18] Beaudet MP. Psychological health - depression. Health Reports 1999; 11: 63-75.

\begin{tabular}{lll}
\hline Received: July 15, 2010 & Revised: September 20, 2010 Accepted: October 10, 2010
\end{tabular}

(C) Patten et al.; Licensee Bentham Open.

This is an open access article licensed under the terms of the Creative Commons Attribution Non-Commercial License (http://creativecommons.org/licenses/by-nc/3.0/) which permits unrestricted, non-commercial use, distribution and reproduction in any medium, provided the work is properly cited. 